\title{
Mitofusin 2 expression dominates over mitofusin 1 exclusively in mouse dorsal root ganglia - a possible explanation for peripheral nervous system involvement in Charcot-Marie-Tooth 2A
}

\author{
Maria Kawalec ${ }^{1}$, Barbara Zabłocka ${ }^{1}$, Dagmara Kabzińska², Jacek Neska ${ }^{1}$, Małgorzata Beręsewicz ${ }^{1}$ \\ ${ }^{1}$ Molecular Biology Unit, Mossakowski Medical Research Centre, Polish Academy of Sciences, Warsaw, ${ }^{2}$ Neuromuscular Unit, \\ Mossakowski Medical Research Centre, Polish Academy of Sciences, Warsaw, Poland
}

\begin{abstract}
Mitofusin 2 (Mfn2), a protein of the mitochondrial outer membrane, is essential for mitochondrial fusion and contributes to the maintenance and operation of the mitochondrial network. Mutations in the mitofusin 2 gene cause axonal Charcot-Marie-Tooth type 2A (CMT2A), an inherited disease affecting peripheral nerve axons. The precise mechanism by which mutations in MFN2 selectively cause the degeneration of long peripheral axons is not known. There is a hypothesis suggesting the involvement of reduced expression of a homologous protein, mitofusin 1 (Mfn1), in the peripheral nervous system, and less effective compensation of defective mitofusin 2 by mitofusin 1. We therefore aimed to perform an analysis of the mitofusin 1 and mitofusin 2 mRNA and protein expression profiles in different mouse tissues, with special attention paid to dorsal root ganglia (DRGs), as parts of the peripheral nervous system. Quantitative measurement relating to $m R N A$ revealed that expression of the Mfn2 gene dominates over Mfn 1 mainly in mouse DRG, as opposed to other nervous system samples and other tissues studied. This result was further supported by Western blot evaluation. Both these sets of data confirm the hypothesis that the cellular consequences of mutations in the mitofusin 2 gene can mostly be manifested in the peripheral nervous system.
\end{abstract}

Key words: mitofusins, peripheral nervous system, CMT2A, Mfn2 mutation manifestation.

\section{Introduction}

Defects in mitochondrial dynamics - mitogenesis, mitophagy and fission/fusion events - are thought to underlie a variety of disorders in humans, such as metabolic diseases (diabetes, hypertension) and neuronal disorders (Parkinson's disease, Alzheimer's disease and Charcot-Marie-Tooth disease [CMT]) [2,4, $7,8,11,16,20]$. One of the earliest indicators of a role for abnormal mitochondrial dynamics in neurological diseases derives from the analysis of genetic mutations leading to CMT, i.e. a group of inherited diseases affecting peripheral nerves [21].

Charcot-Marie-Tooth diseases can be caused by several different mutations in various genes in which an unusual number of mutations occur in mitochondrial-associated proteins, including proteins involved in the regulation of mitochondrial dynamics (e.g. mitofusin 2 [Mfn2] and ganglioside-induced differentiation-associated protein 1 [GDAP1]). For example, 
a large subset of patients with CMT type 2A (CMT2A) have been found to manifest mutations in the mitofusin 2 gene.

While disease-associated mutations in MFN2 are seen to lead to abnormal formation of mitochondrial networks in peripheral nerve axons [14], the precise cellular physiological mechanisms underlying the CMT2A pathology are still poorly understood. One puzzling aspect of CMT2A is that symptoms are restricted to very specific tissues or cell types, despite the fact that mutant MFN2 alleles are expressed in every cell [10]. Hence, one of the hypotheses explaining this problem holds that the axonal neuropathy manifestations of CMT2A could be due to impaired expression of homologous protein - mitofusin 1 (MFN1) - in the peripheral nervous system as compared with other tissues, as well as less effective compensation for defective mitofusin 2 by mitofusin $1[9,10,18]$.

Mitofusins 1 and 2 are mitochondrial GTPases, implicated in the tethering of adjacent outer mitochondrial membranes (OMMs) through the formation of homo- or heterodimers [5]. Murine Mfn1 and Mfn2 are approximately $63 \%$ identical at the amino acid level, and in several contexts they exhibit functional overlap or complementation in the mediation of OMM fusion [23]. However, there is growing evidence to indicate differences in function. Since Mfn 1 knockout has presented more severe aberrations in mitochondrial network formation than Mfn2-deficient cells, Mfn1 is considered to have stronger fusion activity [19]. Moreover, it is suggested that fusion of outer and inner mitochondrial membranes mainly entails Mfn1 [12]. On the other hand, Mfn2, rather than Mfn1, is present on the endoplasmic reticulum (ER), tethering it to mitochondria facilitating calcium flux and autophagy [9,23]. Finally, Mfn2 seems to have some regulatory effect on the cell cycle extending beyond its fusion activity, with impaired expression seemingly correlating with insulin resistance and hyperproliferation in hypertension [23].

In the current study seeking to verify the hypothesis regarding a low level of MFN1 in peripheral nerves as an additional factor responsible for axonal neuropathy manifestation in CMT2A, we aimed to analyse both the mRNA and protein expression profiles regarding $M f n 1$ and 2 in different mouse tissues, paying special attention to the dorsal root ganglia as parts of the peripheral nervous system [12].

\section{Material and methods Animals}

All animal procedures were conducted in accordance with instructions from the Local Commission for Ethics of Experiments on Animals, with every effort being made to minimise animal suffering and the number of animals needed for reliable scientific data to be obtained. Adult C57BL/ 6 mice obtained from the Animal House of the Mossakowski Medical Research Centre, Polish Academy of Sciences, were decapitated under deep anaesthesia, with subsequent dissecting out of the dorsal root ganglia (DRG), cerebral cortex, cerebellum, spinal cord, heart, muscle (quadriceps femoris), liver, and kidney, as well as a fragment of skin. Tissues were frozen and then crushed into a fine powder under liquid nitrogen, portioned for both protein and mRNA expression analysis and stored at $-80^{\circ} \mathrm{C}$ until used. Due to the small amount of material, DRGs were homogenised immediately after dissection, with neither the freezing nor the crushing in liquid nitrogen steps. Hence, in the case of DRGs, at least three mice were used for gene expression studies and another three for protein detection.

\section{Gene expression}

RNA from freshly dissected DRGs was isolated using a Qiagen RNeasy Micro Kit (74004), while isolations from fatty tissues (such as kidney and liver) and other tissues were performed using a Qiagen RNeasy Lipid Tissue Mini Kit (74804) and Qiazol Lysis Reagent (Qiagen, 79306) respectively. RNA samples were subjected to DNase I digestion (Thermo Scientific, EN0521) to remove genomic DNA, and then cleaned using a Thermo Scientific Gene Jet RNA Purification Kit (K0731). For RT-PCR, a First Strand cDNA Synthesis Kit (Thermo Scientific, K1612) was used. Quantitative real-time PCR was then performed, the TaqMan primers and probes (from Life Technologies) being: Gene expression assay Mm00612599_m1 for Mfn1; FAM; Gene expression assay Mm00500120_m1 for Mfn2; FAM; Gene expression assay Mm03928990_g1 for 18s ribosomal RNA; VIC. 18s RNA was used as an internal control. Results were calculated automatically using the $\Delta \Delta \mathrm{Ct}$ method, presented by reference to the relative expression of $M f n 1$ and $M f n 2$, and shown as the $M f n 1 / M f n 2$ ratio \pm standard deviation (Fig. $1 \mathrm{~A}$ ). For particular samples from the nervous system the 

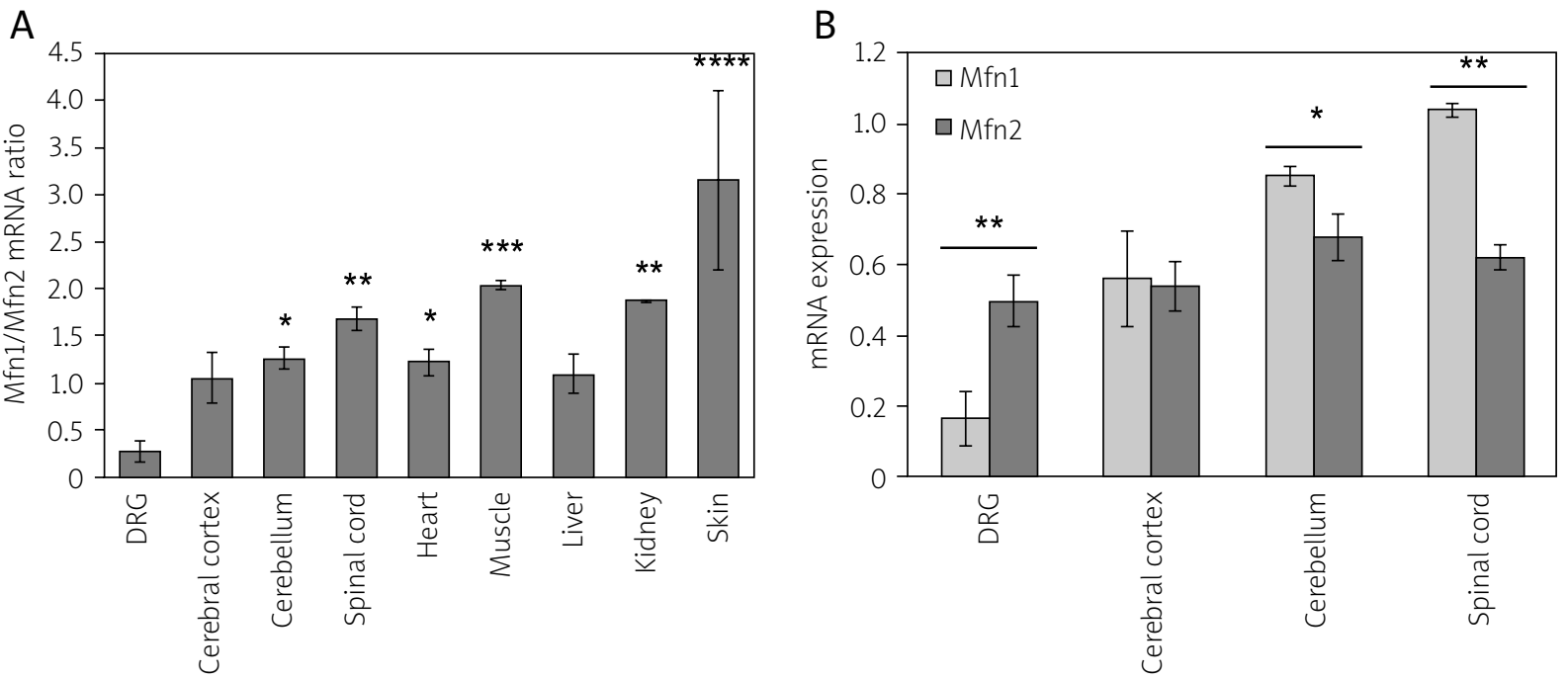

Fig. 1. Comparative analysis of Mfn1 and Mfn2 mRNA expression in different mouse tissue revealed that mitofusin 2 expression only prevails over that of mitofusin 1 in dorsal root ganglia (DRG). In DRG samples the $M f n 1 / M f n 2$ ratio gave an average value of $0.27 \pm 0.08$, indicating $M f n 2$ expression three times greater than that of Mfn1. Real-time PCR results were calculated using the $\Delta \Delta \mathrm{Ct}$ method and are presented (A) as means for the Mfn1 to Mfn2 mRNA ratio \pm standard deviations $\left({ }^{*} p<0.05\right.$, ${ }^{* *} p<0.01$, ${ }^{* *} p<0.001$, ${ }^{* * * *} p<0.0001$ vs. DRG) and (B) as relative values of Mfn 1 and Mfn2 mRNA expression $\left({ }^{\star} p<0.05,{ }^{* *} p<0.01\right)$. Statistical significance was determined using one-way ANOVA with the Bonferroni test in 1A or Student's t-test in $1 \mathrm{~B}$.

relative expression of both Mfn1 and Mfn2 mRNA is presented as mean mRNA expression \pm standard deviation (Fig. 1B).

\section{SDS-PAGE and immunodetection}

Tissue powder and freshly dissected DRGs were homogenized in lysis buffer containing $20 \mathrm{mM}$ Tris$\mathrm{HCl}$ at $\mathrm{pH}$ 7.5, $150 \mathrm{mM} \mathrm{NaCl}, 1 \mathrm{mM} \mathrm{Na}{ }_{2}$ EDTA, $1 \mathrm{mM}$ EGTA, 1\% Triton X-100, $2.5 \mathrm{mM}$ sodium pyrophosphate, $1 \mathrm{mM} \beta$-glycerophosphate, $1 \mathrm{mM} \mathrm{Na}_{3} \mathrm{VO}_{4}, 1 \mu \mathrm{g}$ / $\mathrm{ml}$ leupeptin and $1 \mathrm{mM}$ PMSF (Cell Signalling, 9803). Following homogenisation, samples were sonicated and centrifuged at $14000 \times \mathrm{g}$ at $4^{\circ} \mathrm{C}$ for $10 \mathrm{~min}$ so that clear tissue lysates were obtained. $20 \mu \mathrm{g}$ portions of these were separated by electrophoresis on $10 \%$ SDS-polyacrylamide gels, before being transferred to nitrocellulose membrane (Healthcare Life Sciences, RPN203E GE). Blots were blocked for 1 hour in TBS-T (10 mM Tris- $\mathrm{HCl}, \mathrm{pH} 7.5,150 \mathrm{mM} \mathrm{NaCl}, 0.05 \%$ Tween 20) containing $5 \%$ skimmed milk, and then incubated for 2 hours with primary antibodies against mitofusin 1 (mouse monoclonal) and mitofusin 2 (rabbit polyclonal) (Sigma Aldrich, WH0055669M4, M6319, $1: 500$ and $1: 1000$ respectively), as well as against glyceraldehyde-3-phosphate dehydrogenase (GAPDH,
Chemicon, MAB374, 1 : 1000), to verify the equal loading of protein per lane. Secondary antibodies conjugated with horseradish peroxidase were visualized using a chemiluminescent kit (Bio-Rad 1705061), in line with the manufacturer's protocol. To confirm anti-Mfn 1 and anti-Mfn2 antibody specificity, mouse embryonic fibroblast (MEF) lysates were also loaded onto each gel (wild type MEF [MEFwt] and Mfn1/Mfn2-null MEFs [MEFMfn1-/-Mfn2-/- , as positive and negative controls, respectively). In order to present the results graphically, densitometric analysis of Western blots was performed using Gel Expert Image analysis software (Nucleo Vision). Obtained values for Mfn 1 and Mfn2 were normalized to values of Mfn1 and Mfn2 from wild type MEF respectively and presented in terms of the relative expression of $M f n 1$ versus $M f n 2$, i.e. as the $M f n 1 / M f n 2$ ratio \pm standard deviation.

\section{Statistical analysis}

The statistical significances of Mfn1/Mfn2 ratios were determined using one-way ANOVA with the Bonferroni test for simultaneous analysis of multiple test groups (Figs. 1A and 2B). Student's t-test was used to evaluate the statistical significance of dif- 
ferences between Mfn1 and Mfn2 mRNA expression in particular tissues (Fig. 1B). Data are presented as means \pm standard deviations. The differences are considered significant where $p<0.05$.

\section{Results}

\section{Mitofusin 2 mRNA expression dominates over that of mitofusin 1 in dorsal root ganglia}

Real-time PCR showed that values for the Mfn1 to Mfn2 mRNA ratio were substantially lower in dorsal root ganglia than in other samples from the nervous system (cerebral cortex, cerebellum and spinal cord), as well as other tested organs (heart, muscle, liver, kidney and skin) (Figs. $1 \mathrm{~A}$ and $1 \mathrm{~B}$ ). In the DRG samples the $M f n 1 / M f n 2$ ratio was $0.27 \pm 0.08$, which is to say that expression of Mfn2 was at a level three times higher than that of Mfn1. In contrast, in the cerebral cortex, cerebellum and spinal cord samples, Mfn1/Mfn2 ratios were $1.05 \pm 0.27,1.26 \pm 0.12$ and $1.67 \pm 0.12$ respectively, indicating similar intensities of expression of the two mitofusins in the cerebral cortex, as well as a dominance of Mfn1 over Mfn2 in the cerebellum and spinal cord (Fig. 1A). The Mfn1/ Mfn2 ratios in the cases of non-nervous-system tissues were above 1 , the average values being: heart: $1.22 \pm 0.14$, muscle: $2.04 \pm 0.05$, liver: $1.10 \pm 0.20$, kidney: $1.87 \pm 0.01$ and skin: $3.15 \pm 0.95$. This indicates similar levels of expression of the two mitofusins in heart and liver, and marked dominance of Mfn1 expression in muscle, kidney and skin.

Moreover, comparison of Mfn 1 and Mfn2 mRNA expression - in particular in samples from the nervous system (the DRG, cerebral cortex, cerebellum and spinal cord) - made it clear that Mfn1 expression in the nervous system varies significantly $(p<$ 0.01 DRG vs. cerebral cortex, cerebellum and spinal cord; $p<0.05$ cerebral cortex vs. cerebellum and spinal cord; $p<0.05$ cerebellum vs. spinal cord) - and much more so than that of Mfn2, whose expression was comparable between the samples (the one-way ANOVA followed by the Bonferroni test revealing no significant differences) (Fig. 1B).

\section{Lower protein level of Mfn1 than Mfn2 in dorsal root ganglia}

Western blot evaluation for the two mitofusins suggests differing $M f n 1 / M f n 2$ ratios in various mouse tissues that were consistent with the mRNA expression ratios (Fig. 2). In DRG the protein Mfn1/ $M f n 2$ ratio was $0.87 \pm 0.18$, indicating a lower level of Mfn1 than of Mfn2 there (Fig. 2B). As with the dorsal root ganglia, so in the muscle sample an Mfn1/Mfn2 ratio below 1 was detected, the values being 0.75 \pm 0.04 . In the case of other tissues, the protein Mfn 1 / Mfn2 ratio was found to be equal to or above 1, with average values of: $1.36 \pm 0.04$ for cerebral cortex, $1.30 \pm 0.04$ for cerebellum, $1.30 \pm 0.26$ for spinal cord, $1.27 \pm 0.04$ for heart, $0.93 \pm 0.1$ for liver, 1.02 \pm 0.16 for kidney and $2.12 \pm 0.20$ for skin. Furthermore, in the cases of muscle and skin samples in particular, Western blots revealed an additional, higher molecular weight band detected with the anti-Mfn2 antibody (Fig. 2A).

\section{Discussion}

Mutations in the MFN2 gene cause axonal Charcot-Marie-Tooth type 2A disease affecting long peripheral axons [22]. While it is already well known that disease-associated mutations in the mitofusin 2 gene lead to abnormal formation of mitochondrial networks [10], as well as disrupting mitochondrial transport $[1,13]$ and ER-mitochondrial interactions, the precise pathomechanism of CMT2A has remained poorly understood. One of the still-unsolved aspects of CMT2A is the way in which symptoms of this disease are limited to very specific tissues or cells, despite the fact that the mutant MFN2 alleles are expressed in every cell. It is on this basis that one hypothesis (proposed by Detmer et al.) suggests that it is predominantly peripheral nerves that are affected in CMT2A patients, because they express low levels of MFN1 and rely primarily on MFN2 [10], which is mutated, malfunctioned and insufficient for the proper fusion process among patients with CMT2A. To elucidate this issue further, we analysed the mRNA expression of Mfn 1 and Mfn2 in various mouse tissues, paying special attention to the dorsal root ganglia as parts of the peripheral nervous system. In parallel, Western blot analysis was performed to evaluate the mitofusins protein profile.

Quantitative PCR revealed that values for the Mfn1 to Mfn2 mRNA ratio were substantially lower in DRG than in other tissues. This phenomenon was not observed in other tissues, Mfn1 mRNA being expressed abundantly, especially in spinal cord as well as in muscle, kidney and skin. While a few studies comparing the expression profiles for the two mitofusins in different tissues have been published 


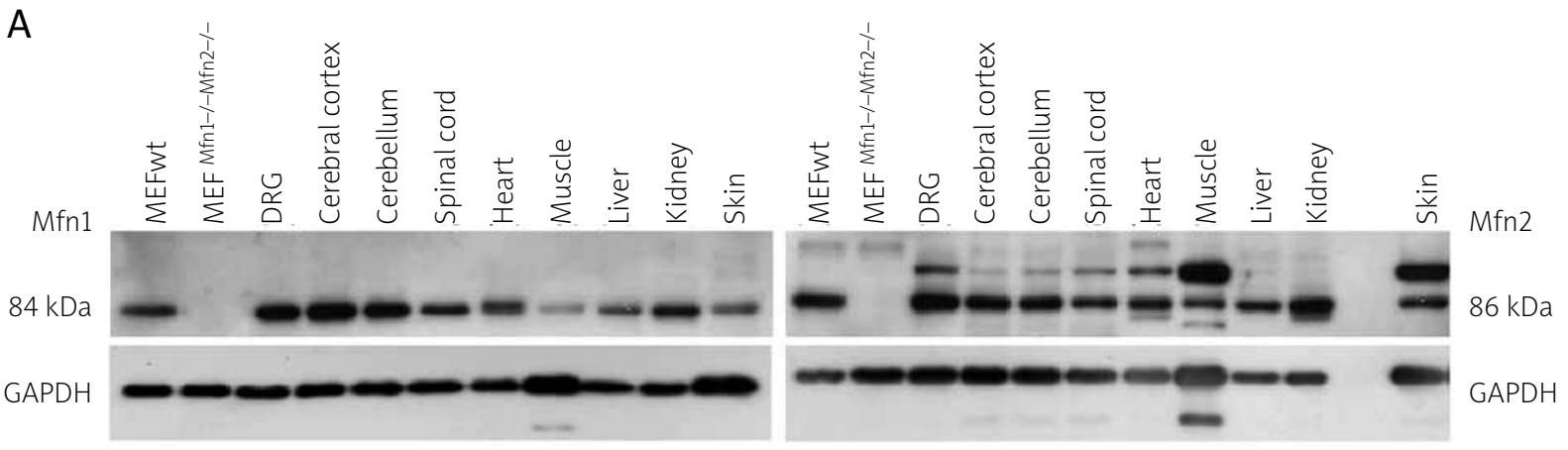

B

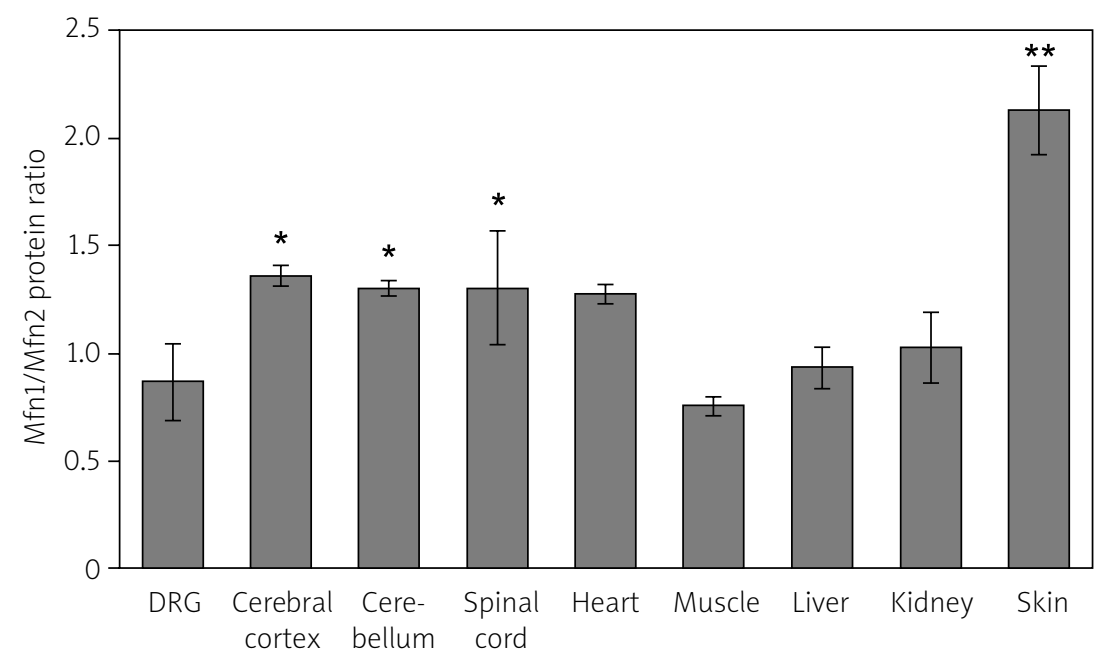

Fig. 2. Comparison of Mfn1 and Mfn2 immunoreactivity suggests a slightly lower level of Mfn1 than Mfn2 in dorsal root ganglia (DRG). A) Tissue lysates $(20 \mu \mathrm{g})$ from different mouse tissue were loaded on $10 \%$ polyacrylamide gel and analysed with anti-Mfn1 (84 kDa) and anti-Mfn2 (86 kDa) antibodies by Western blot. To confirm anti-Mfn1 and anti-Mfn2 antibody specificity, mouse embryonic fibroblast (MEF) lysates were also loaded onto gel (wild type MEF [MEFwt] and Mfn1/Mfn2-null MEFs [MEFMfn1-/-Mfn2-/-]), as positive and negative controls. GAPDH immunoreactivity was assayed for loading control. Immunoblots shown are representative of at least three independent experiments. B) Quantitative analysis of Western blot bands normalized to Mfn1 or Mfn2 in MEFwt and expressed as the relative immunoreactivity of Mfn1 vs. Mfn2 (Mfn1/Mfn2 ratio). Results are presented as means \pm standard deviations; ${ }^{*} p<0.05,{ }^{\star *} p<0.01$ vs. DRG.

previously, these have never included an analysis of the peripheral nervous system [19]. For example, a Northern blot analysis showed that Mfn1 transcripts could be detected at similar levels in a variety of human tissues and were elevated intensely in the heart, while Mfn2 mRNA was expressed abundantly in heart and muscle tissue, but only present at low levels in many other tissues [19]. In addition, Cao et al. (2013) detected the presence of equal amounts of the two mitofusins in rat spinal cord [3].

To recap, the data for mRNA expression offer quite firm evidence of axonal damage to peripheral nerves in CMT2A being due to inadequate compen- sation for dysfunctional MFN2 by MFN1, which is fully functional, but not expressed at a sufficient level.

In our study, the comparative evaluation of Mfn 1 and Mfn2 protein expression in different mouse tissues suggests lower protein expression for Mfn1 as compared with Mfn2 in the DRG, as well as levels of mitofusin 1 higher than those of mitofusin 2 in the cerebral cortex, cerebellum and spinal cord. Similarly, Mfn1 seems to dominate over Mfn2 in skin and heart tissue. Unexpectedly, the estimated ratio of Mfn1/Mfn2 proteins in muscle is seen to be as low as in the dorsal root ganglia, suggesting a higher level of Mfn2 protein there. The significance of this 
observation is unclear, but it is tempting to speculate that over-expression of Mfn 1 mRNA is not fully translated to protein, or regulated in a special manner. It is worth noting that in the case of muscle tissue an additional, higher than $86 \mathrm{kDa}$, dense band was detected in the Western blot by the anti-Mfn2 antibody. A similar band pattern is visible in the skin sample, which may suggest muscle-cell content or a similar modification pattern for Mfn2 protein in various cell types. A parallel fine band is also visible in DRG and heart samples, which suggests a common level for the two forms of Mfn2 protein. It has been reported that mitofusin 2 binds to MARCH-V - mitochondrial ubiquitin ligase - and may possibly undergo ubiquitylation [15]. There are also data regarding $\mathrm{Mfn} 2$ phosphorylation [6]. These post-translational modifications increase molecular mass and can alter cellular localisation and protein function. It is possible that Mfn2 plays additional roles in skeletal muscle cells, e.g. in calcium signalling, trafficking or selective mitophagic elimination of damaged mitochondria, as was described recently for heart tissue, through mechanisms requiring the mitochondrial kinase PINK1 and the cytosolic ubiquitin ligase Parkin [6]. Similarly, it has been reported that Mfn1 may be a ubiquitylation substrate, undergoing modification that in this case is necessary for proper mitochondrial network formation [17].

Although the progress in understanding the molecular machinery and role of mitofusins in mitochondrial dynamics and the regulation of mitochondrial metabolism is notable, several issues regarding mitofusin 2 function and its relationship with mitofusin 1 remain poorly understood. First, the extent to which there is complementation among mitofusins is unclear. Are they functionally interchangeable? Second, the correlation between mitochondria and neuromuscular junction formation and function remains unknown. The existence of unresolved questions of this magnitude points to the significance of the topic and the necessity for additional studies.

\section{Acknowledgments} 74640.

The project was supported by NSC grant NN 4024

\section{Disclosure}

Authors report no conflict of interest.

\section{References}

1. Baloh RH, Schmidt RE, Pestronk A, Milbrandt J. Altered axonal mitochondrial transport in the pathogenesis of Charcot-MarieTooth disease from mitofusin 2 mutations. J Neurosci 2007; 27: 422-430.

2. Beal MF. Mitochondria take center stage in aging and neurodegeneration. Ann Neurol 2005; 58: 495-505.

3. Cao Y, Lv G, Wang YS, Fan ZK, Bi YL, Zhao L, Guo ZP. Mitochondrial fusion and fission after spinal sacord injury in rats. Brain Res 2013; 1522: 59-66.

4. Chan PH. Mitochondria and neuronal death/survival signaling pathways in cerebral ischemia. Neurochem Res 2004; 29: 19431949.

5. Chen H, Detmer SA, Ewald AJ, Griffin EE, Fraser SE, Chan DC. Mitofusins Mfn1 and Mfn2 coordinately regulate mitochondrial fusion and are essential for embryonic development. J Cell Biol 2003; 160: 189-200.

6. Chen Y, Dorn GW. PINK1-phosphorylated mitofusin 2 is a Parkin receptor for culling damaged mitochondria. Science 2013; 340: 471-475.

7. Cho DH, Nakamura T, Lipton SA. Mitochondrial dynamics in cell death and neurodegeneration. Cell Mol Life Sci 2010; 67: 34353447.

8. de Brito OM, Scorrano L. Mitofusin 2: a mitochondria-shaping protein with signaling roles beyond fusion. Antioxid Redox Signal 2008; 10: 621-633.

9. Del Bo R, Moggio M, Rango M, Bonato S, D’Angelo MG, Ghezzi S, Airoldi G, Bassi MT, Guglieri M, Napoli L, Lamperti C, Corti S, Federico A, Bresolin N, Comi GP. Mutated mitofusin 2 presents with intrafamilial variability and brain mitochondrial dysfunction. Neurology 2008; 71: 1959-1966.

10. Detmer SA, Chan DC. Complementation between mouse Mfn1 and Mfn2 protects mitochondrial fusion defects caused by CMT2A disease mutations. J Cell Biol 2007; 176: 405-414.

11. Fujimoto $M$, Hayashi T. New insights into the role of mitochondria-associated endoplasmic reticulum membrane. Int Rev Cell Mol Biol 2011; 292: 73-117.

12. Melli G, Hoke A. Dorsal Root Ganglia Sensory Neuronal Cultures: a tool for drug discovery for peripheral neuropathies. Expert Opin Drug Discov 2009; 4: 1035-1045.

13. Misko A, Jiang S, Wegorzewska I, Milbrandt J, Baloh RH. Mitofusin 2 is necessary for transport of axonal mitochondria and interacts with the Miro/Milton complex. J Neurosci 2010; 30: 4232-4240.

14. Misko AL, Sasaki Y, Tuck E, Milbrandt J, Baloh RH. Mitofusin2 mutations disrupt axonal mitochondrial positioning and promote axon degeneration. J Neurosci 2012; 32: 4145-4155.

15. Nakamura N, Kimura Y, Tokuda M, Honda S, Hirose S. MARCH-V is a novel mitofusin 2- and Drp1-binding protein able to change mitochondrial morphology. EMBO Rep 2006; 7: 1019-1022.

16. Oettinghaus B, Licci M, Scorrano L, Frank S. Less than perfect divorces: dysregulated mitochondrial fission and neurodegeneration. Acta Neuropathol 2012; 123: 189-203.

17. Park YY, Lee S, Karbowski M, Neutzner A, Youle RJ, Cho H. Loss of MARCH5 mitochondrial E3 ubiquitin ligase induces cellular senescence through dynamin-related protein 1 and mitofusin 1 . J Cell Sci 2010; 123: 619-626. 
18. Rouzier C, Bannwarth S, Chaussenot A, Chevrollier A, Verschueren A, Bonello-Palot N, Fragaki K, Cano A, Pouget J, Pellissier JF, Procaccio V, Chabrol B, Paquis-Flucklinger V. The MFN2 gene is responsible for mitochondrial DNA instability and optic atrophy 'plus' phenotype. Brain 2012; 135: 23-34.

19. Santel A, Frank S, Gaume B, Herrler M, Youle RJ, Fuller MT. Mitofusin-1 protein is a generally expressed mediator of mitochondrial fusion in mammalian cells. J Cell Sci 2003; 116: 2763-2774.

20. Schon EA, Przedborski S. Mitochondria: the next (neurode)generation. Neuron 2011; 70: 1033-1053.

21. Stetler AR, Leak RK, Gao Y, Chen J. The dynamics of the mitochondrial organelle as a potential therapeutic target. Cereb Blood Flow Metab 2013; 33: 22-32.

22. Verhoeven K, Claeys KG, Zuchner S, Schroder JM, Weis J, Ceuterick C, Jordanova A, Nelis E, De Vriendt E, Van Hul M, Seeman P, Mazanec R, Saifi GM, Szigeti K, Mancias P, Butler IJ, Kochanski A, Ryniewicz B, De Bleecker J, Van den BP, Verellen C, Van Coster R, Goemans N, Auer-Grumbach M, Robberecht W, Milic R, V, Nevo Y, Tournev I, Guergueltcheva V, Roelens F, Vieregge P, Vinci P, Moreno MT, Christen HJ, Shy ME, Lupski JR, Vance JM, De Jonghe P, Timmerman V. MFN2 mutation distribution and genotype/phenotype correlation in Charcot-Marie-Tooth type 2. Brain 2006; 129: 2093-2102.

23. Zorzano A, Liesa M, Sebastian D, Segales J, Palacin M. Mitochondrial fusion proteins: Dual regulators of morphology and metabolism. Semin Cell Dev Biol 2010; 21: 566-574. 